\title{
Optimization of dual-saturation single bolus acquisition for quantitative cardiac perfusion and myocardial blood flow maps
}

Javier Sánchez-González ${ }^{*}$, Rodrigo Fernandez-Jiménez ${ }^{2,3}$, Nils D Nothnagel ${ }^{1}$, Gonzalo López-Martín², Valentin Fuster ${ }^{2,4}$ and Borja Ibañez $2,3^{*}$

\begin{abstract}
Background: In-vivo quantification of cardiac perfusion is of great research and clinical value. The dual-bolus strategy is universally used in clinical protocols but has known limitations. The dual-saturation acquisition strategy has been proposed as a more accurate alternative, but has not been validated across the wide range of perfusion rates encountered clinically. Dual-saturation acquisition also lacks a clinically-applicable procedure for optimizing parameter selection. Here we present a comprehensive validation study of dual-saturation strategy in vitro and in vivo.

Methods: The impact of saturation time and profile ordering in acquisitions was systematically analyzed in a phantom consisting of 15 tubes containing different concentrations of contrast agent. In-vivo experiments in healthy pigs were conducted to evaluate the effect of $\mathrm{R}^{*}$ on the definition of the arterial input function (AIF) and to evaluate the relationship between $\mathrm{R}^{*}$ and $\mathrm{R} 1$ variations during first-pass of the contrast agent. Quantification by dual-saturation perfusion was compared with the reference-standard dual-bolus strategy in 11 pigs with different grades of myocardial perfusion.

Results: Adequate flow estimation by the dual-saturation strategy is achieved with myocardial tissue saturation times around $100 \mathrm{~ms}$ (always $<30 \mathrm{~ms}$ of AIF), with the lowest echo time, and following a signal model for contrast conversion that takes into account the residual R2* effect and profile ordering. There was a good correlation and agreement between myocardial perfusion quantitation by dual-saturation and dual-bolus techniques $\left(R^{2}=0.92\right.$, mean difference of $0.1 \mathrm{ml} / \mathrm{min} / \mathrm{g}$; myocardial perfusion ranges between 0.18 and $3.93 \mathrm{ml} / \mathrm{min} / \mathrm{g}$ ).
\end{abstract}

Conclusions: The dual-saturation acquisition strategy produces accurate estimates of absolute myocardial perfusion in vivo. The procedure presented here can be applied with minimal interference in standard clinical procedures.

Keywords: Dual saturation acquisition strategy, Absolute quantitative cardiac perfusion, Cardiovascular magnetic resonance

\section{Background}

Myocardial perfusion is affected by several pathological situations, and the degree of myocardial hypo-perfusion correlates with clinical prognosis [1-3]. There is a need for safe noninvasive techniques that accurately quantify absolute myocardial perfusion. Cardiovascular magnetic resonance (CMR) has been proposed as a noninvasive radiation-free

\footnotetext{
*Correspondence: Javier.Sanchez.Gonzalez@philips.com; Blbanez@cnic.es 'Philips Healthcare Iberia, Maria de Portugal 1. 28050, Madrid, Spain ${ }^{2}$ Centro Nacional de Investigaciones Cardiovasculares (CNIC), Melchor Fernandez Almagro 3. 28029, Madrid, Spain

Full list of author information is available at the end of the article
}

imaging tool for absolute quantification of myocardial perfusion, and its performance compares well with positron emission tomography (PET) [4].

Semi-quantitative methods based on the contrast enhancement ratio $[5,6]$ or upslope index $[7,8]$ have been proposed as a means of obtaining quantitative results from first-pass MR images; however, these approaches systematically underestimate myocardial perfusion reserve (MPR) compared with fully quantitative methods [9]. Fully quantitative measurement of absolute myocardial perfusion has been achieved with a dualbolus protocol [10], in which a dilute bolus of contrast- 
agent is injected at the beginning of the acquisition, followed by a full-concentration bolus of the same volume [11]. The dilute bolus ensures a subsaturating concentration of contrast agent in the left ventricle during measurement of contrast enhancement in the blood pool (arterial input function, AIF). The second bolus, of undiluted contrast agent, allows accurate estimation of contrast uptake by the myocardium. This method has been validated against microspheres in an animal model, showing a good correlation across low, normal, and hyperemic myocardial blood flow (MBF) [10], and a patient study showed good agreement with MPR estimates by PET [4]. However, clinical implementation of the dualbolus method is not trivial because of the many manipulations required [11,12], limiting its inclusion in clinical routine. Moreover, the delay between bolus injections means that it is not possible to ensure acquisition of AIF and muscle information in the same cardiac situation.

An alternative approach is dual-saturation acquisition, in which AIF and myocardial tissue information are collected after injection of a single bolus of undiluted contrast agent [13]. In this approach, signal saturation during AIF definition is avoided by using a short saturation time, and myocardial tissue information is subsequently obtained with longer saturation times $[13,14]$. MBF estimation by this method requires careful control of three parameters: saturation times for accurate evaluation of contrast enhancement in the blood pool and cardiac muscle; R2* effects, especially during AIF estimation [15]; and the influence of $\mathrm{k}$-space ordering during high resolution image read-out [16].

The purpose of this study was to define a clinically useful procedure for accurate quantification of myocardial perfusion using the dual-saturation strategy. Using state-of-the-art 3Tesla-CMR, we analyzed the influence of saturation time (TS), echo time (TE), contrast injection rate, and image profile ordering on estimates of AIF and myocardial contrast uptake in a set of in-vitro (phantom) and in-vivo (large animal model) experiments (Figure 1). Signal acquisition and modeling derived from the in vitro analysis were incorporated in an in vivo comparison of the dual-bolus and dual-saturation strategies over a wide range of perfusion values, including hypo-perfusion (post-infarct), normal, and hyperemic MBF (pharmacological hyperemia), revealing generation of accurate MBF maps by dualsaturation CMR.

\section{Methods}

All imaging experiments were performed on a 3T-TX Achieva platform (Philips Healthcare, The Netherlands) equipped with a 32-channel phased-array cardiac coil.

\section{Phantom experiments}

The phantom consisted of 15 plastic $50 \mathrm{ml}$ falcon tubes, each filled with distilled water and a specific concentration of contrast agent gadolinium diethylenetriamine pentaacetic

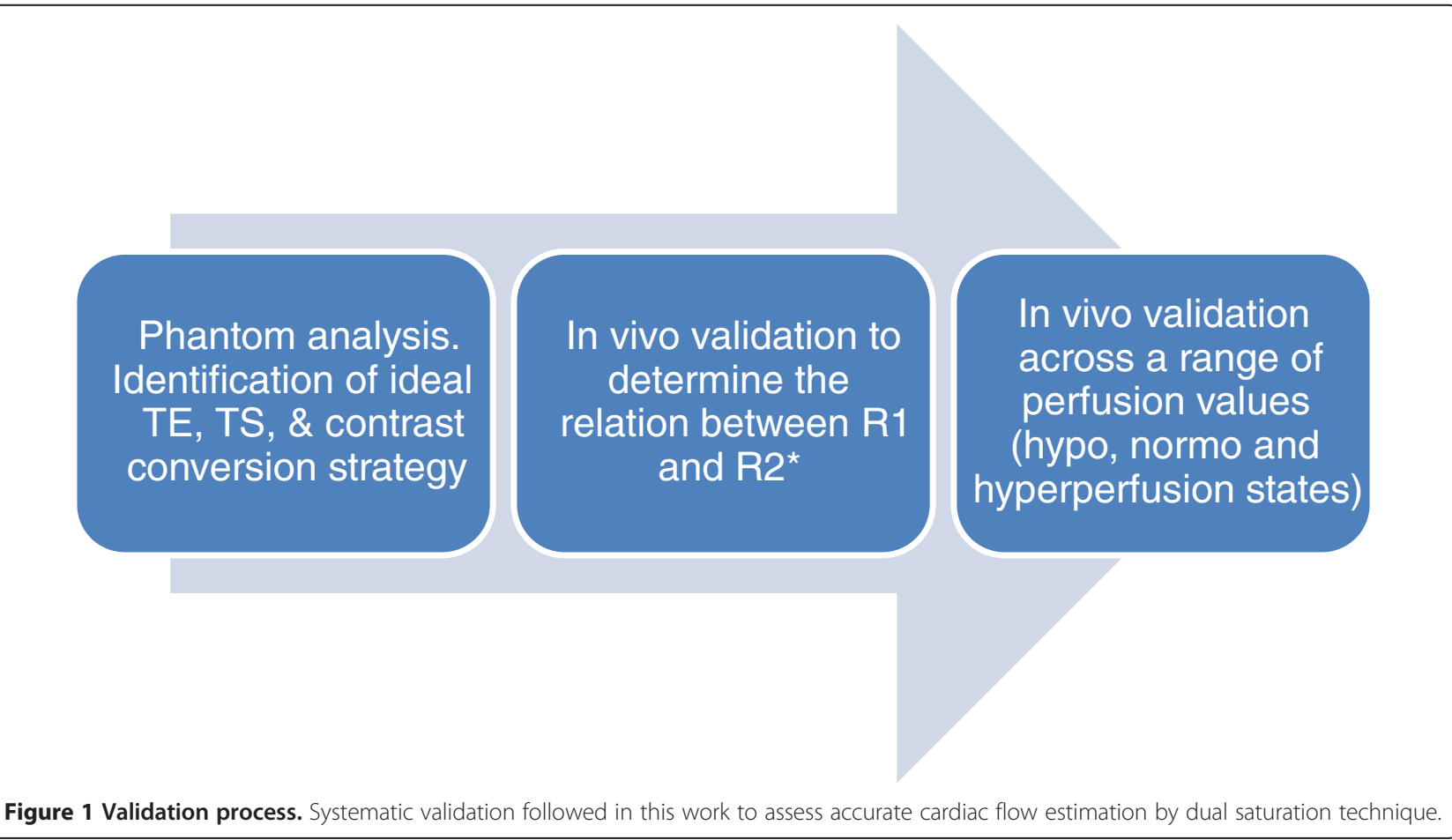


acid (Gd-DTPA, Magnevist, Schering AG, Berlin, Germany), ranging from 0 to $22.5 \mathrm{mM}$. Three imaging experiments were performed with the same phantom to evaluate the effect of contrast concentration on R1 values and saturation times, and the influence of profile ordering on estimated final contrast concentration.

\section{Estimation of $R 1$ values at different contrast concentrations} Gold-standard T1 values of each tube were estimated using a look-locker inversion recovery acquisition with 147 inversion times ranging from $6.06-5886.06 \mathrm{~ms}$, with a time interval of $40 \mathrm{~ms}$. A new inversion pulse was applied every $10 \mathrm{~s}$ to avoid signal saturation. To reduce the influence of excitation readout at different inversion times, an excitation flip angle of $5^{\circ}$ was applied during the TFE shot [17]. For tubes with R1 higher $20 \mathrm{~Hz}$, a second sequence was applied with lower TR and FA (TR/ $\mathrm{FA}=3 \mathrm{~ms} / 2^{\circ}$ ). In both cases the final R1 values were estimated by fitting the signal intensity at different inversion times to the previously described signal model [17].

\section{Evaluation of saturation times at different contrast concentrations}

The second experiment was designed to evaluate the signal behavior at different saturation times and contrast concentrations. All parameters except TS were held constant (single shot TFE, TR/TE/FA $=2.26 \mathrm{~ms} / 1.07 \mathrm{~ms} / 15^{\circ}$ ), and ten images of each tube were acquired at TS $=10,20$, 30, 40, 50, 60, 70, 80, 90 and $200 \mathrm{~ms}$. An additional proton density $\left(\mathrm{S}_{\mathrm{PD}}\right)$ image was acquired without saturation pulse to normalize the signal intensity of each contrast concentration $\left(\mathrm{S} / \mathrm{S}_{\mathrm{PD}}\right)$. All images were acquired by low-high profile ordering to enable short TS and to avoid read-out effects on signal intensity.

\section{Evaluation of the influence of profile ordering on estimated final contrast concentration}

The third phantom experiment was designed to evaluate the influence of profile ordering on the conversion of MR signal changes into contrast concentration variations. This experiment was performed with 13 of the tubes with fixed TS of $100 \mathrm{~ms}$. The read-out sequence was based on single-shot spoiled TFE acquisition (TR/ TE/FA/TFEshot $=1.95 \mathrm{~ms} / 0.9 \mathrm{~ms} / 15^{\circ} / 55$ shots $)$ with halfscan factors of $65 \%$ and linear and reverse-linear profile ordering. For each tube, we also computed signal intensity with the signal model described in the Appendix, using different $\mathrm{T} 1$ values and the same acquisition parameters in order to allow later comparison with real acquired values.

\section{Animal experiments}

Experimental procedures were performed in castratedmale Large-White pigs. The study protocol was approved by the Institutional Animal Research Committee and conducted in accordance with recommendations of the Guide for the Care and Use of Laboratory Animals.

Pigs were sedated by intramuscular injection of ketamine $(20 \mathrm{mg} / \mathrm{kg})$, xylazine $(2 \mathrm{mg} / \mathrm{kg})$, and midazolam $(0.5 \mathrm{mg} / \mathrm{kg})$. A marginal vein in the ear was cannulated for peripheral intravenous access. Sedation was maintained by a continuous intravenous infusion of ketamine $(2 \mathrm{mg} / \mathrm{kg} / \mathrm{h})$, xylazine $(0.2 \mathrm{mg} / \mathrm{kg} / \mathrm{h})$ and midazolam $(0.2 \mathrm{mg} / \mathrm{kg} / \mathrm{h})$.

The first in-vivo experiment was designed to evaluate the R1 and R2* variations at different contrast concentrations during first-pass perfusion, in order to establish the relationship between the two relaxation rates invivo. In a second in-vivo experiment, 11 pigs with different degrees of myocardial perfusion were examined simultaneously by the dual-bolus and dual-saturation methods to allow comparison of cardiac perfusion estimates by the two approaches. For hypoperfusion, 6 pigs underwent a closed-chest transmural myocardial infarction (45 minutes ischemia in the left anterior descending coronary artery followed by reperfusion); the procedure was performed with a dedicated percutaneous catheter inserted via the femoral artery [18,19]. Index CMR was performed 45 days post-infarction. For regional hyperemia, 4 pigs were subjected to intracoronary infusion with sodium nitroprusiate $(4 \mathrm{mg} / \mathrm{kg})$ or papaverine $(0.5 \mathrm{mg} / \mathrm{kg})$ during image acquisition inside the MRI scanner. Normoperfusion was assessed in 1 healthy animal undergoing CMR.

\section{In-vivo image acquisition}

\section{Evaluation of $R 1$ and $R 2 *$ changes at different contrast} concentrations during first-pass perfusion

To define the relation between $\mathrm{R} 1$ and $\mathrm{R} 2 *$ values and contrast concentration in-vivo, different TS and TE were acquired during first pass of the contrast agent. To estimate R1 values every second, 6 saturation times were acquired over the range from $3.2-328.2 \mathrm{~ms}$, with intervening gaps of $64.8 \mathrm{~ms}$. The saturation times were acquired by sharing the same saturation pulse between 6 slice locations in transverse orientation along the descending aorta. The slices were acquired in ascending order to ensure that a saturated blood signal due to RF excitation pulses in one slice did not influence the signal acquired from the other slices. To estimate R2* values for every TS, four TE were acquired over the range from 0.8-3.8 ms with intervening gaps of $1.0 \mathrm{~ms}$. The same experiment was performed dynamically every second over one minute, during which $0.1 \mathrm{mmol} / \mathrm{kg}$ of $\mathrm{Gd}$ DTPA Magnevist (Schering AG, Berlin, Germany) was injected at a rate of $3 \mathrm{ml} / \mathrm{s}$, followed by a $20 \mathrm{ml}$ saline flush.

For these experiments, $\mathrm{R} 2$ * values were obtained by fitting the signal at different TE to a mono-exponential 
model. The same procedure was applied for all TE values at different TS, and the mean was taken as the final result. $R 1$ values were obtained by fitting the signal from different TS at the shortest TE to a regular saturation recovery model. After estimation of R2* and R1 for each dynamic acquisition, the values of the two quantities were fitted to a quadratic relation, as previously proposed [20].

\section{Comparison between dual-bolus and dual-saturation methods}

To evaluate the two quantification strategies, dual-saturation acquisition was combined with the dual-bolus injection protocol. For dual-bolus injection, the injection protocol was done using a single power MR injector Spectris Solaris (Medrad, Warrendale, Pennsylvania) using a previously described methodology by Ishida et al. [11]. Two boluses of Gd-DTPA Magnevist
(Schering AG, Berlin, Germany) of equal volume and different concentration $(0.01 \mathrm{mmol} / \mathrm{kg}$ and $0.1 \mathrm{mmol} / \mathrm{kg})$ were injected at $3 \mathrm{ml} / \mathrm{s}$, each followed by a $20-\mathrm{ml}$ saline flush. A waiting time of $25 \mathrm{~s}$ was established between injections to ensure proper insulation of both curves during image analysis.

Three high resolution images (voxel size $2.6 \times 2$. $6 \times 10.0 \mathrm{~mm}^{3}$ ) and an additional interleaved low resolution image $\left(6.8 \times 2.6 \times 10.0 \mathrm{~mm}^{3}\right)$ were acquired at every R-R interval over $120 \mathrm{~s}$. Interleaved low resolution image was acquired between the saturation pulse of the first high resolution image and image readout, sharing the same saturation pulse between low and high resolution images. To prevent any signal interaction between both images the planning was defined avoiding crosses between slices. When the heart rate was above $120 \mathrm{bpm}$ one high resolution slice was removed from a

\section{AIF Interleave Sequence details}

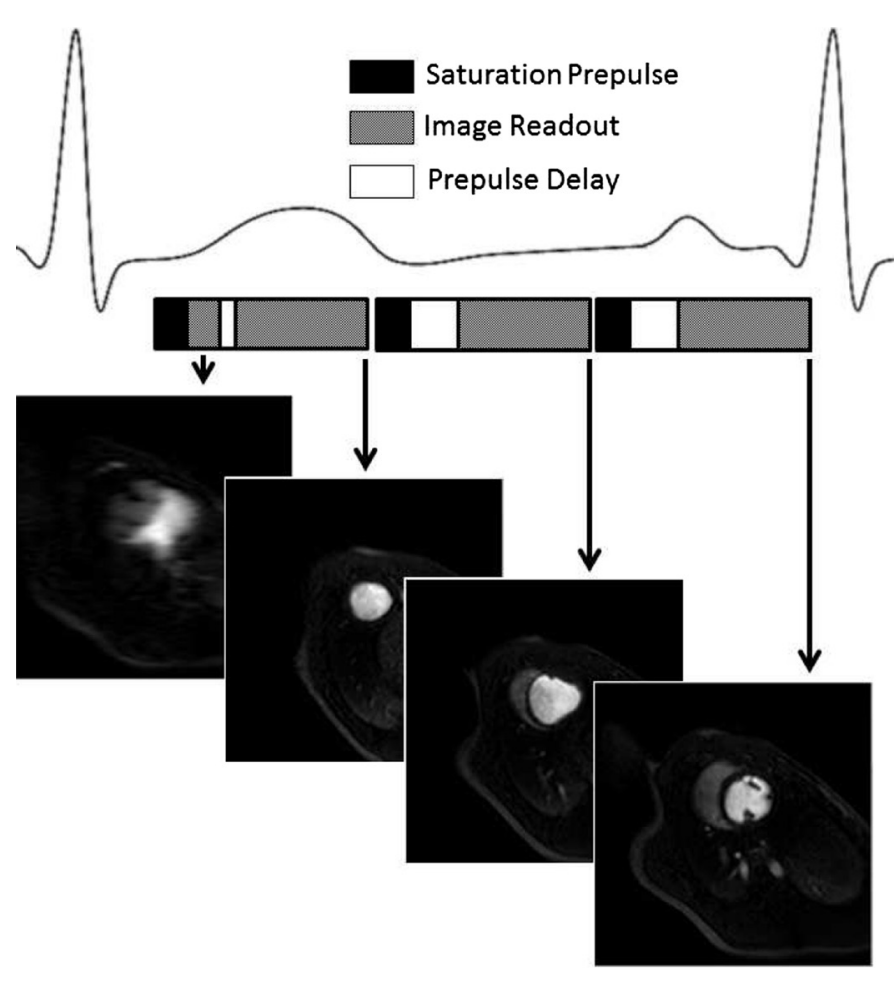

b

\section{AIF Selection}

Dual Saturation

Dual Bolus

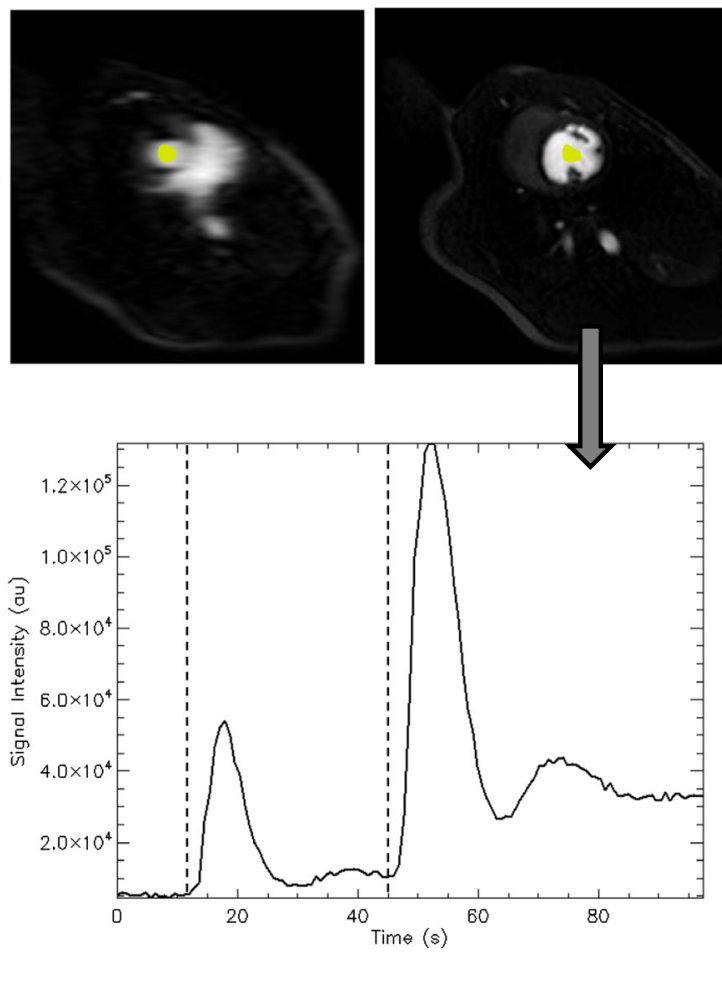

Figure 2 Sequence Details and AIF drawing for Low and High resolution images. a) represents the sequence details showing the short saturation time is interleaved acquired sharing the saturation pulse with the first high resolution image. b) ROI example for AlF selection in the dual saturation and dual bolus cases and an example of the signal-intensity curve at the high resolution. Dotted vertical lines represent the selection of the limits for diluted AIF. 
the acquisition to include all slices in a single RR interval. The image sequence consisted of spoiled TFE acquisition with a saturation time of $100 \mathrm{~ms}$ for high resolution images and $20 \mathrm{~ms}$ for low resolution images $\left(\mathrm{TR} / \mathrm{TE} / \mathrm{FA}=2.0 \mathrm{~ms} / 0.9 \mathrm{~ms} / 15^{\circ}\right)$. Images were acquired with reverse-linear profile ordering with a half scan factor of $75 \%$ and a SENSE factor of 2.6. All perfusion images were reconstructed to $2.0 \times 2.0 \mathrm{~mm}^{2}$ in-plane resolution. To obtain baseline T1 values, an additional MOLLI (modified look-locker inversion recovery) T1 map was acquired with a 3-5 scheme [21] at the same location before every perfusion scan.

The full image analysis to obtain flow information was performed using custom software written in IDL8.1 (Exelis Visual Information Solutions, Boulder, Colorado). Time-intensity curves were transformed into concentration curves using the methodology described in the Appendix for low and high resolution images and both perfusion strategies. In dual-bolus analysis AIF was defined by drawing a region of interest (ROI) in the left ventricle cavity of high resolution images and manually choosing the region of the time curve corresponding to the first diluted contrast as the portion of the curve between the first and second upslope shoulders of the intensity curve (Figure 2b). The duration of the final AIF was established as the time difference between both temporal positions and the initial point was established at the upslope shoulder of the full concentration injection (dashed lines in Figure 2b). After signal cropping the diluted AIF was temporary registered to the full contrast AIF to prevent shift with the muscle contrast uptake by automatic method that find the maximum correlation between both curves by temporal shifting of diluted AIF. Finally, the diluted, cropped and registered AIF was scaled according to the concentration difference. In dual-saturation analysis an ROI was drawn on low resolution images with no scaling factor and using the whole time curve in the deconvolution process. Model-based deconvolution was used to estimate flow, using an exponential model with 2 fitting parameters:

$$
\mathrm{h}(\mathrm{t})=\mathrm{F} \exp [-\mathrm{kt}]
$$

where $\mathrm{F}$ represents the magnitude of the function, directly related to the blood flow, and $\mathrm{k}$ describes the decay rate of $\mathrm{h}(\mathrm{t})$ due to contrast wash-out. No shift was included in the model since AIF and cardiac muscle contrast uptake were measured close enough, making such correction unnecessary. Cardiac flow was assessed by scaling the $\mathrm{F}$ value according to a muscle density of $1.05 \mathrm{~g} / \mathrm{ml}$ and assuming a constant hematocrit of 0.35 following high permeability model

$$
M B F=\frac{F\left[\mathrm{~min}^{-1}\right]}{(1-\text { Hematocrit }) * 1.05[\mathrm{~g} / \mathrm{ml}]}
$$

After flow-map generation, a quantitative comparison of the dual-bolus and dual-saturation methods was performed by ROI analysis in the ischemic or hyperemic region and the contralateral wall for all acquired slices and animals. The ROI was defined in the dual-bolus flow maps and was copied to dual-saturation maps to extract the final numerical data. Correlation and Bland-Altman plot analysis were performed to compare flow measured with the two approaches.

\section{Results}

\section{Phantom experiments}

Estimation of $T 1$ values at different contrast concentrations

Table 1 shows the T1 and R1values of the different contrast concentration tubes. These values were taken as the reference T1 values to assess signal behavior at different contrast concentrations in subsequent phantom experiments. The $\mathrm{T} 1$ values ranged from $3693 \mathrm{~ms}$ for the phantom without contrast agent to $14 \mathrm{~ms}$ for a contrast concentration of $22 \mathrm{mM}$. This contrast concentration corresponds to the upper limit observed in human experiments with a $0.1 \mathrm{mmol} / \mathrm{kg}$ contrast injection at a rate of $6 \mathrm{ml} / \mathrm{s}$ [22].

\section{Evaluation of saturation times at different contrast concentrations}

The effect of the TS on signal intensity at different contrast concentrations is shown in Figure 3. For a TS of $10 \mathrm{~ms}$, signal $\left(\mathrm{S} / \mathrm{S}_{\mathrm{PD}}\right)$ increased linearly with contrast concentration in the phantom, with a linear fitting correlation coefficient of $\mathrm{R} 2=0.986$. In contrast, for a TS

Table $1 \mathrm{~T} 1$ and R1 values of the phantom tubes

\begin{tabular}{ll}
\hline $\mathbf{T 1} \mathbf{( m s )}$ & $\mathbf{R 1}(\mathbf{H z})$ \\
\hline 3693 & 0.27 \\
2685 & 0.37 \\
641 & 1.56 \\
581 & 1.72 \\
451 & 2.22 \\
400 & 2.50 \\
335 & 2.98 \\
304 & 3.29 \\
255 & 3.92 \\
208 & 4.80 \\
138 & 7.22 \\
70 & 14.31 \\
34 & 29.01 \\
21 & 48.71 \\
14 & 71.69 \\
\hline
\end{tabular}




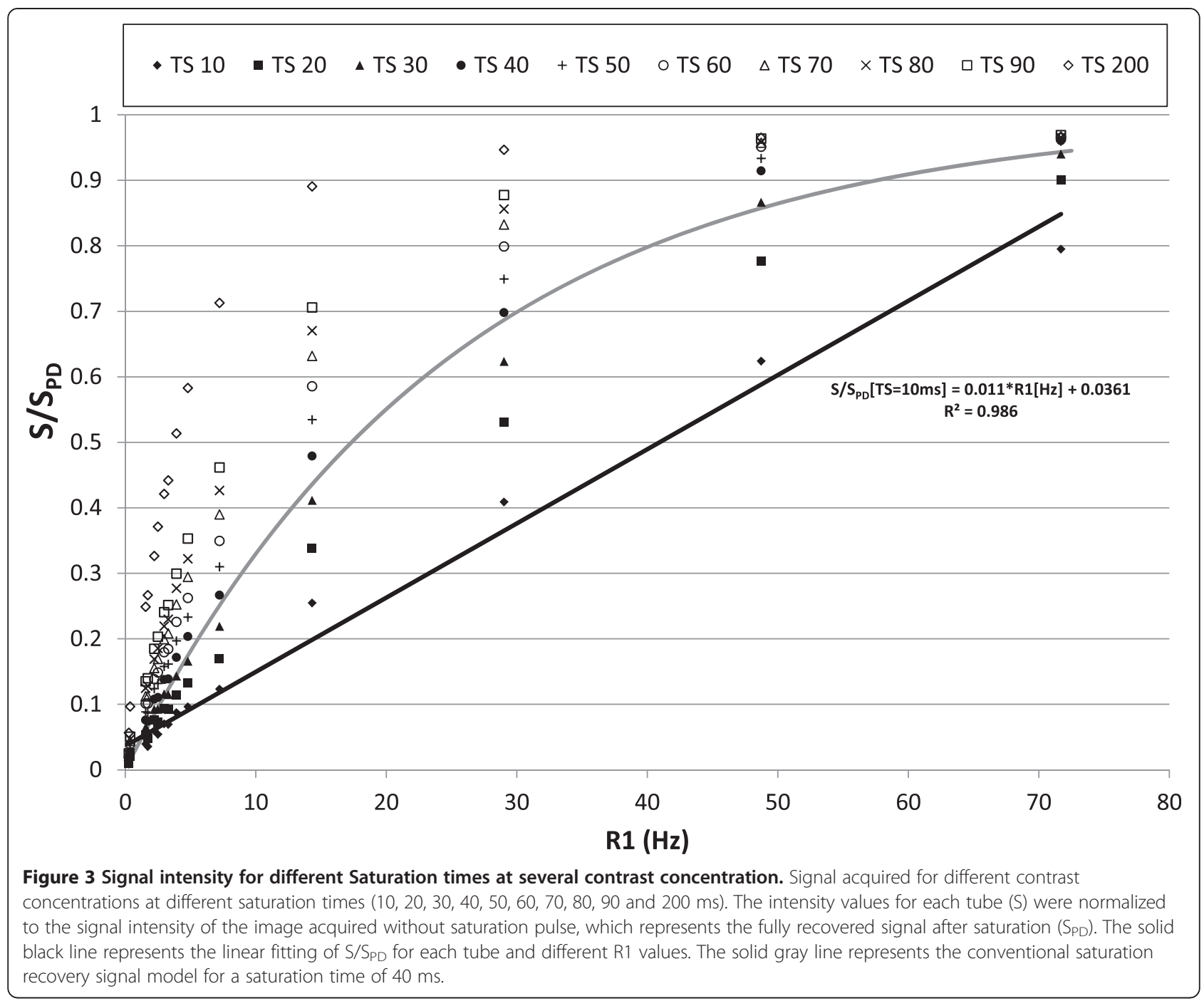

above $40 \mathrm{~ms}$ the signal was fully recovered at higher contrast concentrations, with a value of $\mathrm{S} / \mathrm{S}_{\mathrm{PD}}$ close to 1 , saturating the signal response.

\section{Evaluation of profile ordering in estimates of final contrast concentration}

The effect of different readout strategies on the signal intensity ratio at different contrast concentrations is represented in Figure 4. Signal intensity ratios were calculated by dividing the measured signal intensity of each tube by the measured signal of the tube filled with pure water, as described in the Appendix. Linear correlation analysis between the measured $\mathrm{S}_{\mathrm{c}} / \mathrm{S}_{\mathrm{b}}$ data (points in Figure 4) and the signal model (solid lines) yielded correlation coefficients of $R^{2}=0.999$ and $R^{2}=0.998$ for reverse-linear and linear profile ordering, respectively. The slopes between the model and the measured data were 0.979 and 0.978 for reverse-linear and linear profile ordering, respectively.

\section{In vivo data}

Evaluation of $R 1$ and $R 2 *$ changes at different contrast concentrations during first-pass perfusion

MR signal changes due to R1 and R2* effects during the first-pass of bolus injection are shown in Figure 5. For longer TE (Figure 5a) there is a drop in signal due to R2* effects at very high contrast concentration. The effect of $\mathrm{R} 2 *$ changes $(\triangle \mathrm{R} 2 *)$ reached a maximum value of $39 \mathrm{~Hz}$ (Figure 5c). Figure 5b shows the effect on the MR signal acquired with different TS during the same bolus injection. For shorter TS the MR signal perfectly follows the contrast bolus, whereas for longer TS the MR signal is fully relaxed, reaching a plateau that limits the ability of the MR signal to track the highest contrast concentrations. The R1 values obtained after fitting the saturation recovery experiment for each time domain point were normalized to those obtained before contrast injection $\left(\Delta R_{1}\right)$ (Figure $5 d$ ).

The relation between $\Delta \mathrm{R}_{2}{ }^{*}$ and $\Delta \mathrm{R}_{1}$ (Figure 6) was fitted to a quadratic relation [23], yielding a correlation 


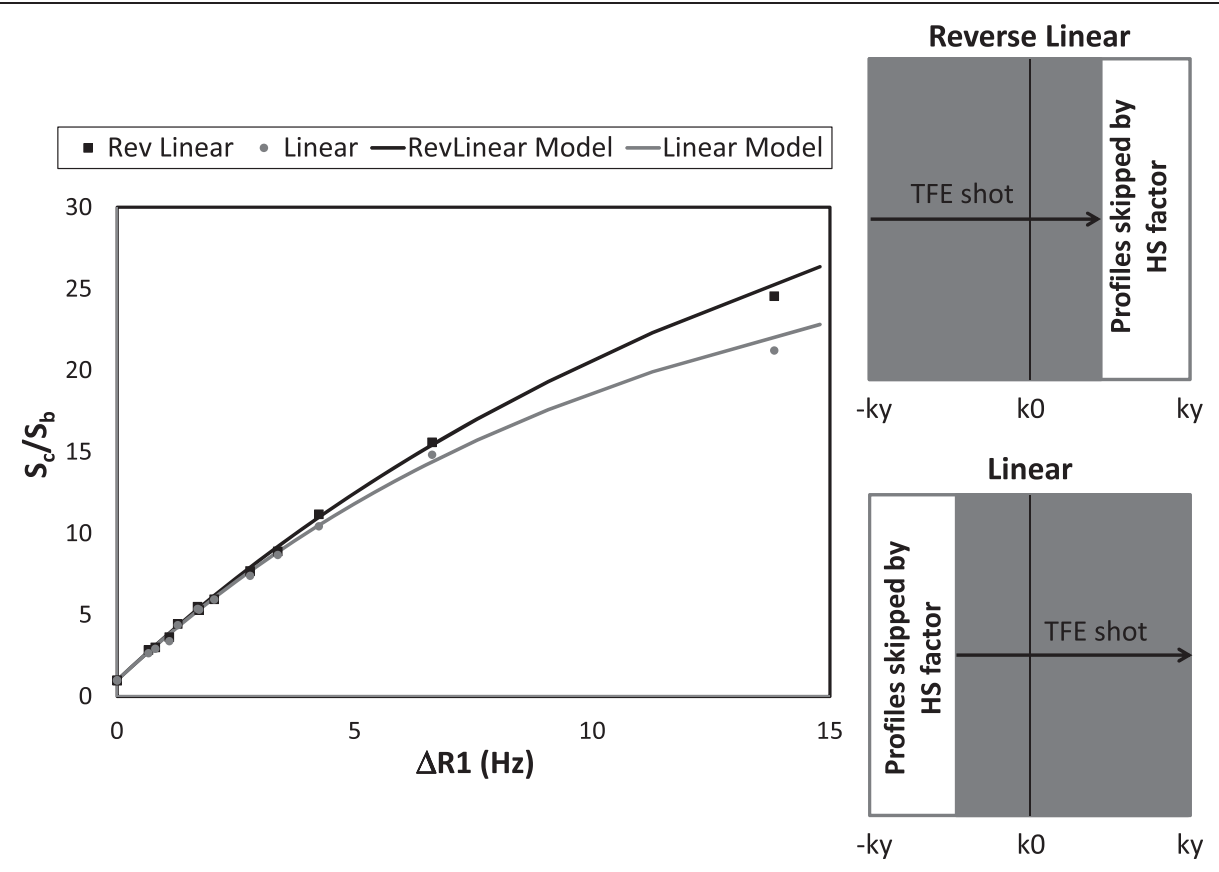

Figure 4 Read-out effects on signal intensity. The graph on the left represents the signal ratio between the signal of the phantoms at different contrast concentrations and the signal of the tube without contrast for Reverse Linear (black squares) and Linear (gray circles) profile ordering. Dotted lines represent the result of the same ratio derived from the signal model described in the appendix for both profile ordering schemes. Solid lines represent the result of the signal model of $S_{c} / S_{b}$ described in the Appendix for both readout strategies.

coefficient of $\mathrm{R}^{2}=0.95$ with a final relation between both quantities as follows:

$$
\Delta \mathrm{R}_{2}^{*}=0.0332 \Delta \mathrm{R}_{1}^{2}+0.165 \Delta \mathrm{R}_{1}-0.0003
$$

The relation established in this equation between $\Delta \mathrm{R} 1$ and $\Delta R 2 *$ effects was subsequently used for contrast estimation in vivo.

\section{Correlation between the dual-bolus and dual-saturation methods}

Representative MBF maps from both techniques are shown in Figure 7 for post infarction left anterior descending coronary artery (Figure 7a) and induced hyperemia of the right coronary artery (Figure 7b). Flow measurements obtained with the two methods (Figure 8) show a good linear relationship $\left(R^{2}=0.92\right)$. Bland-Altmann plot (Figure 9) shows a small disparity of $0.1 \mathrm{ml} / \mathrm{min} / \mathrm{g}$ between the two perfusion measurements.

\section{Discussion}

In this study we present a validation analysis for myocardial perfusion by dual-saturation acquisition including in vitro and in vivo experiments for the most important parameters affecting the quantification of absolute cardiac perfusion. The in vitro results were included in a comparison with the well-established dual-bolus technique in a pig model over a wide range of perfusion values. Our data show that a TS below $30 \mathrm{~ms}$ is sufficient to avoid AIF saturation. Although a low TE is desirable to avoid R2* effects on AIF estimation, we have also described the relation between $\mathrm{R} 1$ and $\mathrm{R} 2{ }^{*}$ during bolus injection and included this in the conversion from signal to contrast concentration. The dual-saturation methodology was tested at flow values ranging from 0.12 to $3.92 \mathrm{ml} / \mathrm{min} / \mathrm{g}$, yielding a correlation coefficient with dual-bolus acquisition close to $1\left(R^{2}=0.92\right)$.

This correlation is slightly higher than that previously reported for analysis of the dual-bolus method with the multiple saturation recovery times (m-SRT) technique [24] (0.92 vs 0.82), probably due to the wider flow range explored in the present study. Moreover, overestimation of m-SRT quantitative perfusion has been reported at low perfusion rates [24]. In our study, measurements with the dual-bolus and dual-saturation methodologies show good agreement at low flow rates, with a constant level close to $0(0.0048 \mathrm{ml} / \mathrm{min} / \mathrm{g})$. This discrepancy with the previous report can be explained by the use of higher contrast concentration in the present study. Direct comparison of contrast concentration between the two reports is difficult due to the use of different contrast agents (Gd-DPTA here and Gd-BOPTA in the previous report); however, here we use a fivefold higher contrast volume for in-vivo experiments (0.1 vs $0.02 \mathrm{mmol} / \mathrm{kg}$ ), while the $\mathrm{r} 1$ relaxivity of Gd-BOPTA is just 1.31 times higher than that of Gd-DPTA [25]. 


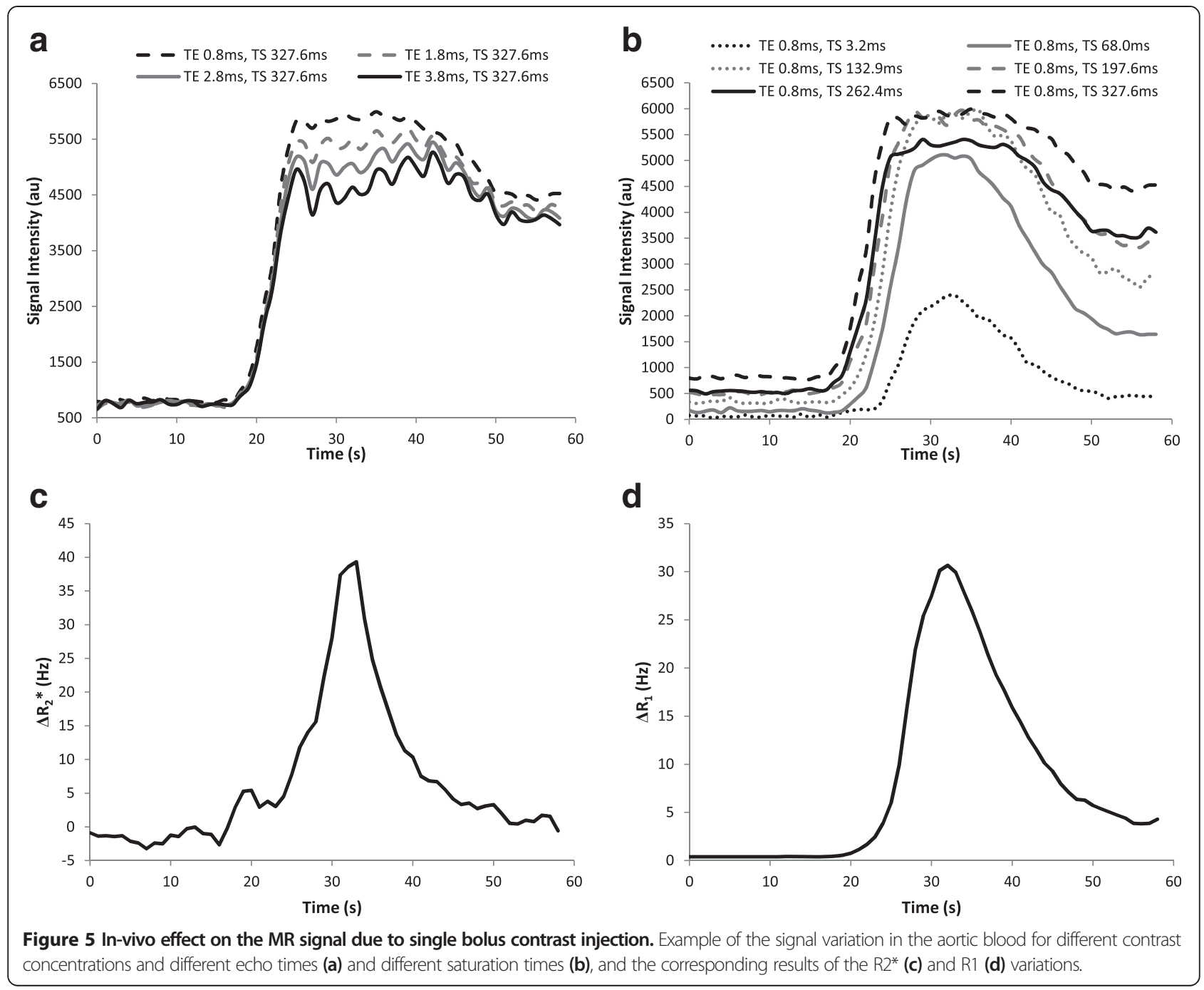

Compared with the dual-bolus method, the dualsaturation approach underestimates flow at high flow rates, a result in agreement with previous findings [24]. This effect can probably be explained by physiological changes to the AIF in the dual-bolus approach at the high contrast concentration and under stress conditions, where it can be more difficult to maintain a constant and homogeneous vasodilation. In contrast, these changes are not seen when a single injection of contrast agent is used since AIF and cardiac muscle information are acquired at the same time.

The Bland-Altman plot showed that the mean difference between the two methods is $0.1 \mathrm{ml} / \mathrm{min} / \mathrm{g}$, which is the minimum flow detected by both techniques and is thus within the expected intrinsic error. The difference increases at higher myocardial flows, probably due to a higher heterogeneity of flow distribution in the cardiac muscle.

In order to obtain reliable perfusion measurements it is necessary to consider different potential factors that can influence estimation of contrast concentration changes in the blood pool (AIF) and cardiac muscle from the MR signal. One factor is a high contrast concentration passing through the ventricular cavity, which directly produces an underestimate of AIF due to over-long TS selection and R2* signal decay. A second factor is the readout effect of the cardiac muscle MR signal in high resolution images and its influence on the estimation of contrast concentration from the MR signal.

Accurate estimation of AIF first requires careful selection of the TS in order to avoid signal saturation at a too high contrast concentration. The results presented in Figure 3 show that in images acquired with TS values below or equal to $30 \mathrm{~ms}$ there is no substantial saturation effect on the MR signal for a maximum R1 of $71.69 \mathrm{~Hz}$. Based on previously reported relaxivity values of Magnevist at $3 \mathrm{~T}$ [23], this value corresponds to a contrast concentration of $21.8 \mathrm{mM}$ in aqueous solution $(\Gamma=3.29 \mathrm{~Hz} / \mathrm{mM}$ at $3 \mathrm{~T})$ and $19.97 \mathrm{mM}$ in blood $(\Gamma=$ $3.59 \mathrm{~Hz} / \mathrm{mM}$ at $3 \mathrm{~T}$ ). This is within the range of previous reported values for contrast concentration in the left ventricle cavity of healthy volunteers receiving an 


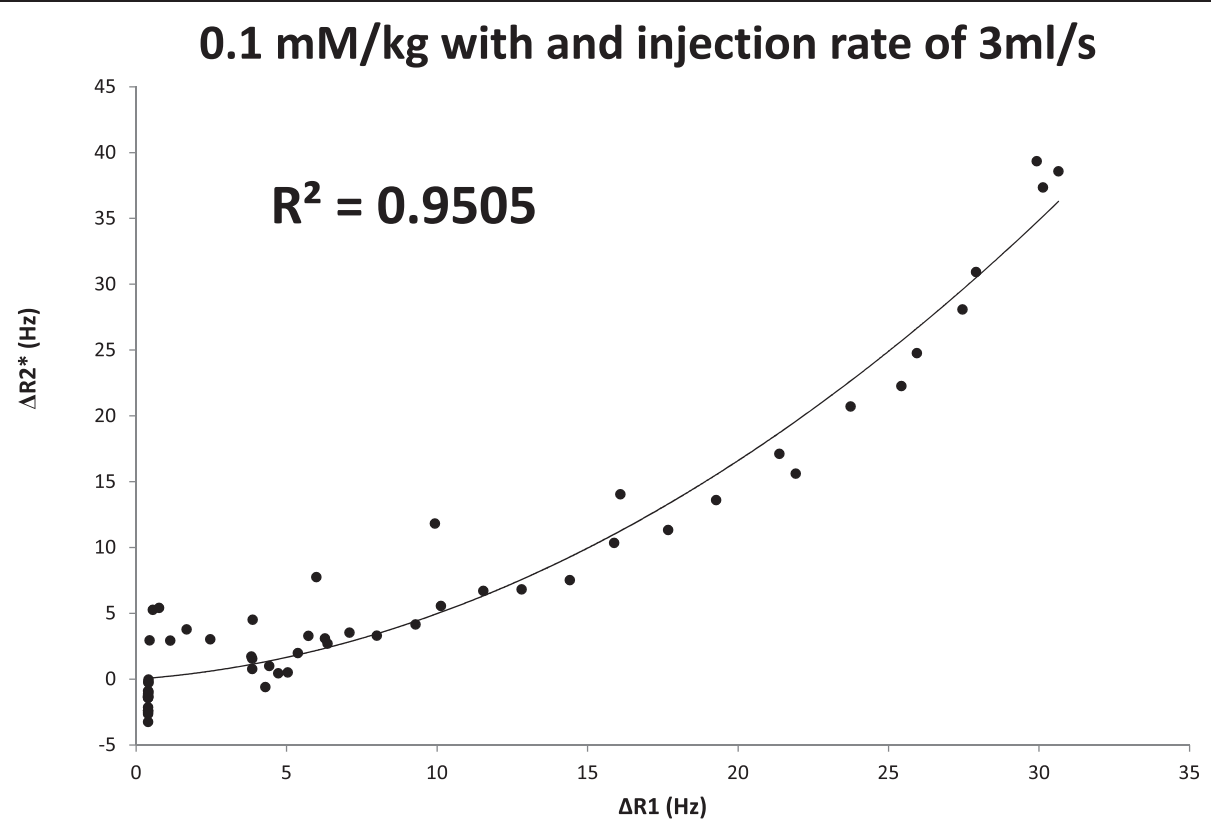

Figure 6 Correlation between R1 and R2* during same in-vivo bolus injection. Relation between the R2* variation and R1 variation obtained from Figure 5 for all dynamics (black dots) and the quadratic fitting between both quantities.

injection of Magnevist $(0.1 \mathrm{mmol} / \mathrm{kg})$ at a rate of $6 \mathrm{ml} / \mathrm{s}$ [16]. The figure also shows that a $200 \mathrm{~ms}$ TS allowed a more detailed analysis of contrast concentrations below 4.24 $\mathrm{mM}(\mathrm{R} 1=13.95 \mathrm{~Hz})$ using the full signal recovery range for this small contrast concentration window [6]. Unfortunately, at longer saturation times it is more difficult to acquire all slices in a single heartbeat, making it necessary to use shorter saturation times.

The second source of error that can affect AIF estimation at high contrast concentration is the potential for R2* effects. This effect can be observed in Figure 5a. During the same contrast injection different echoes are acquired with the corresponding $\mathrm{R} 2 *$ signal decay. With a contrast dose of $0.1 \mathrm{mmol} / \mathrm{kg}$ injected at a rate of $3 \mathrm{ml} / \mathrm{s}$ the maximum $\Delta \mathrm{R} 2^{*}$ value was $39 \mathrm{~Hz}$, which underestimated AIF by $3.5 \%$ for the $\mathrm{TE}$ used in the present study ( $\mathrm{TE}=0.9 \mathrm{~ms}$ ) and by $2.2 \%$ for previously reported TE $(\mathrm{TE}=0.58 \mathrm{~ms}$ ) [26]. This effect has been compensated for in the contrast conversion procedure by using the estimated relation between the R1 and R2* effects (Figure 4) in contrast conversion curves. To be able to estimate this relation, R1 and $\mathrm{R} 2 *$ were estimated simultaneously during the same contrast injection. Assuming a linear relation between contrast concentration and R1 changes [23], a quadratic a

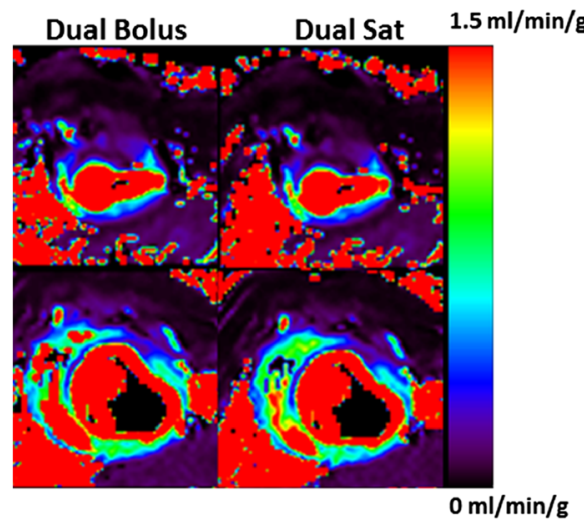

b

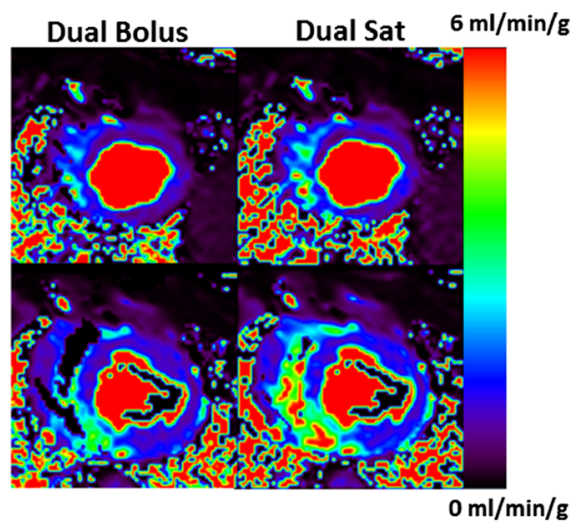

Figure 7 Representative Myocardial Blood Flow maps. Representative flow maps for left anterior descendant (LDA) coronary occlusion (a) and selected hyperemia at right coronary artery (RCA) (b) estimated with dual-bolus and dual-saturation strategies. In both cases dual-bolus and dual-saturation maps have equalized scales. 


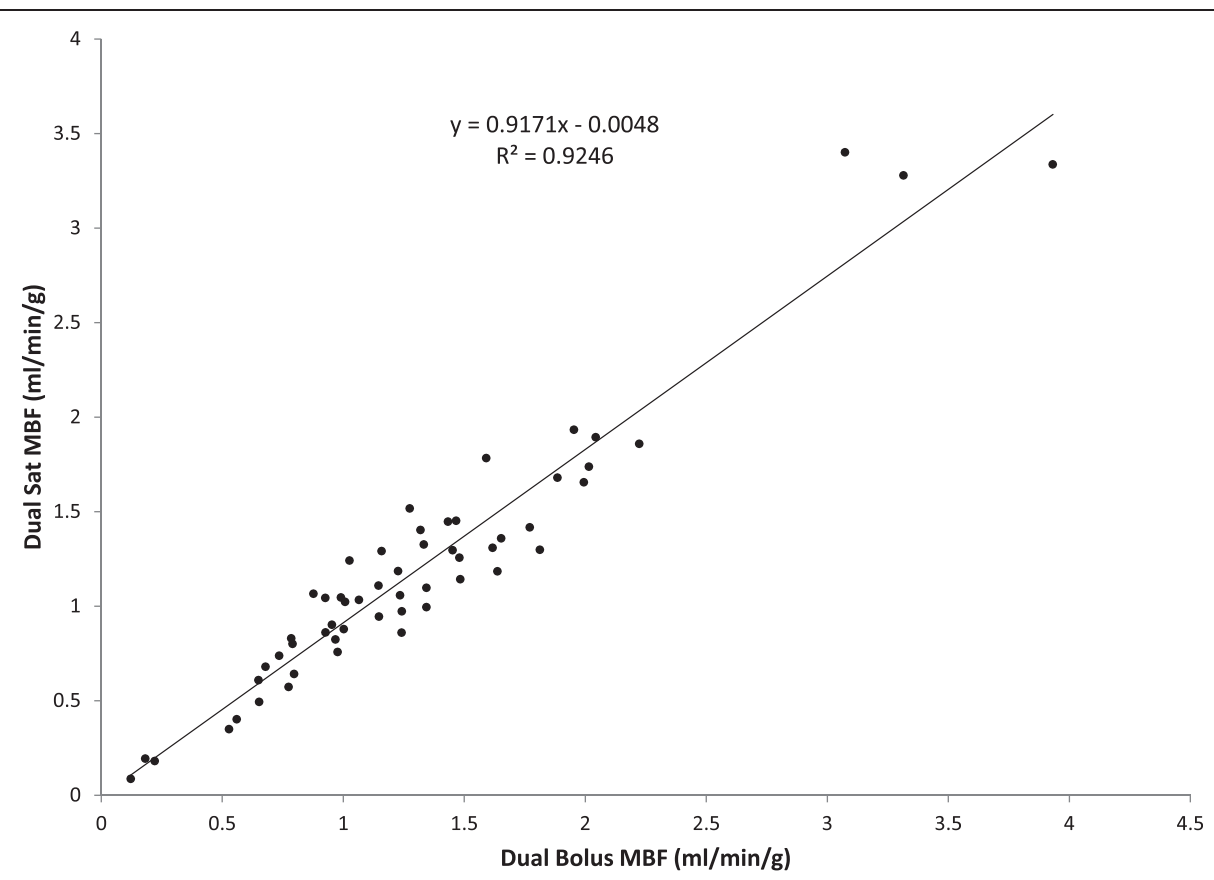

Figure 8 Correlation analysis between Dual Bolus and Dual Saturation MBF. Scatter plot and linear regression fitting (solid line) comparing the quantitative results obtained with the dual-bolus and dual-saturation approaches (black dots).

relation was found between $\mathrm{R} 1$ and $\mathrm{R} 2 *$ values in-vivo, as previously reported $[15,22]$.

Readout effects are more pronounced in high resolution images than in the AIF estimation due to the application of higher turbo factors before reaching the center of the k-space. This series of RF pulses influences magnetization and therefore the estimation of contrast concentration within the cardiac muscle. The proposed signal model demonstrates a good agreement with the acquired data, yielding a correlation coefficient close to 1 .

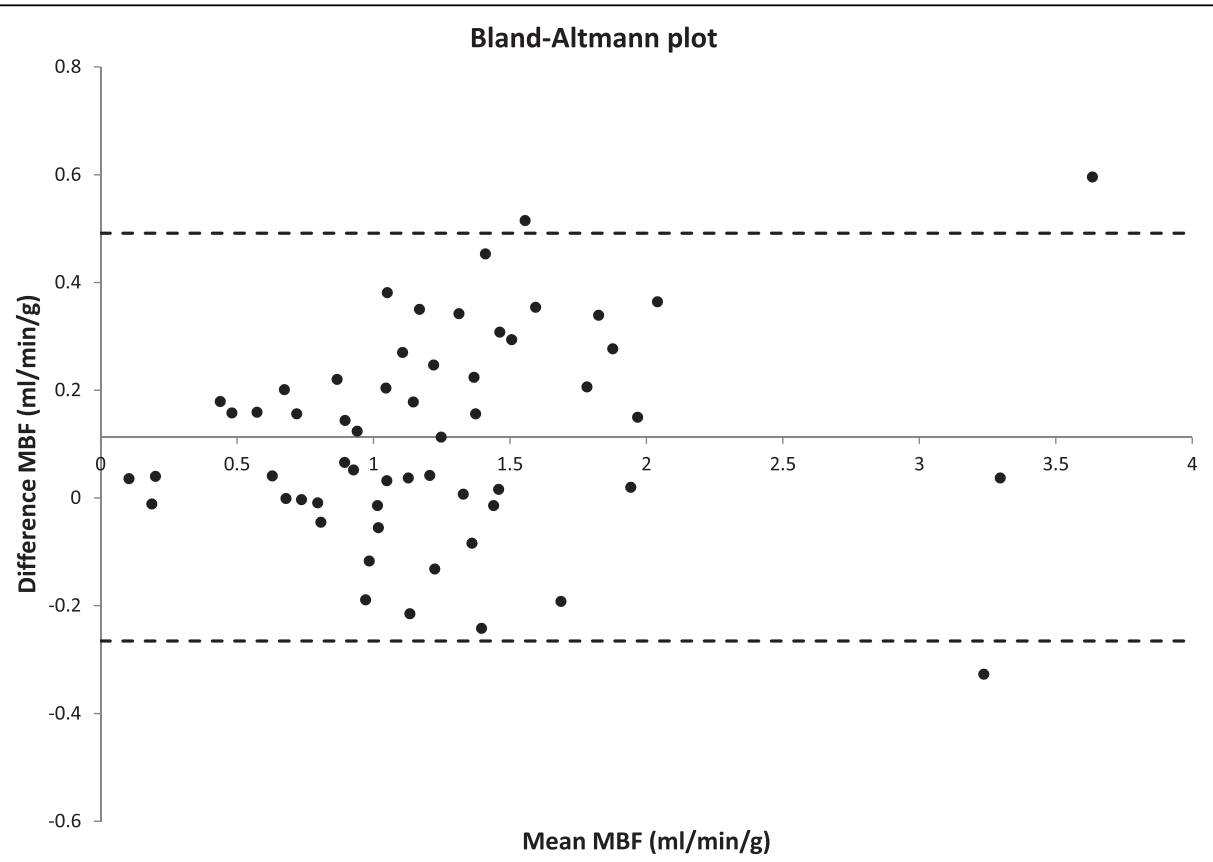

Figure 9 Bias plot between Dual Bolus and Dual Saturation MBF. Bland-Altman plot comparing the quantitative results obtained with the dual-bolus and dual-saturation approaches. 


\section{Limitations}

The main limitation of the present study is that the dual-saturation method was compared to dual-bolus acquisition as a gold standard. Comparison with microspheres was not performed. However, the dual-bolus method has consistently shown good agreement in phantom analysis [27], with microspheres [10,28], and in nuclear medicine in human studies [4].

\section{Conclusions}

The dual-saturation strategy for absolute quantification of myocardial perfusion by 3T-CMR shows an almost perfect correlation with the dual-bolus method across a wide range of perfusion scenarios (form post-infarction hypoperfusion to pharmacologically-induced hyperemic status). The dual-saturation strategy is moreover easier to implement in clinical protocols. Obtaining reliable flow measurements with the dual-saturation strategy requires careful selection of specific parameters. Chief among these are the correct selection of TS to ensure adequate evaluation of contrast uptake in the AIF (TS < $30 \mathrm{~ms}$ ) and cardiac muscle ( $\mathrm{TS}<100 \mathrm{~ms}$ ); the relation between the contrast injection protocol and the R2* effects on the AIF-always attempting to acquire images with the shortest possible TE; and the readout scheme for proper conversion of signal intensity changes into real contrast uptake.

\section{Appendix}

For the contrast concentration used in clinical practice, a linear relationship can be assumed between the changes in contrast concentration and R1 variation in tissues. The first step to assess contrast concentration changes is the accurate evaluation of $\mathrm{R} 1$ changes $(\Delta \mathrm{R} 1)$ as the difference between the tissue $R 1_{c}$ after contrast injection and the baseline $R 1_{b}$ value. Accurate estimates of $R 1$ variation changes are therefore required for a proper estimation of contrast concentration.

Conventional cardiac perfusion acquisitions are based on a saturation recovery experiment followed by different readout strategies normally based on acquisition techniques for single-shot spoiled turbo field echo (TFE) or hybrid spoiled TFE echo planar imaging (TFE-EPI). TFE acquisition is normally desired since shorter echo times are achievable, thus making this approach less sensitive to R2* [29]. The signal model described in this report can be applied to both TFE and TFE-EPI.

To obtain reliable $\mathrm{R} 1$ changes it is necessary to have a detailed understanding of the progress of magnetization between the saturation pulse and the center of the kspace $(\mathrm{k} 0)$, where the contrast of the image is generated. In saturation recovery experiment (Figure 2) the MR signal recovers after the saturation pulse with the corresponding T1 value according to equation [A1]

$$
\mathrm{S}_{\mathrm{T} 0}=\mathrm{S}_{0} \mathrm{e}^{-R 2_{b}^{*} T E}\left(1-e^{-\mathrm{R} 1_{\mathrm{b}} \mathrm{T} 0}\right),
$$

where $\mathrm{T} 0$ is the time between the saturation pulse and the starting point of the image readout; $R 1_{b}$ and $S_{0}$ are $R 1$ and the fully recovered signal intensity of the tissue; TE is the sequence echo time; and $\mathrm{R} 2{ }_{\mathrm{b}}{ }^{*}$ represents the transverse relaxation rate. After T0 the imaging readout sequence starts with different excitation pulses that affect the spin magnetization before reaching the center of the $\mathrm{k}$-space, where the image contrast is defined. These signal changes can be expressed as:

$$
\mathrm{S}_{\mathrm{b}}=\mathrm{e}^{-R 2_{b}^{*} T E}\left[\mathrm{~S}_{0}^{*}-\left(\mathrm{S}_{0}^{*}-\mathrm{S}_{\mathrm{T} 0}\right) \mathrm{e}^{-R 1_{b}^{*}(\mathrm{TS}-\mathrm{T} 0)}\right]
$$

where TS is the defined saturation time in the acquisition sequence, and $R 1_{b}^{*}$ and $S_{0}^{*}$ are the modified $\mathrm{R} 1$ and steady-state magnetization of a spoiled gradient echo acquisition, which are related to original $\mathrm{T} 1$ and $\mathrm{S}_{0}$ values by:

$$
\epsilon=\frac{\mathrm{S}_{0}^{*}}{\mathrm{~S}_{0}}=\frac{\mathrm{R}_{1}}{R_{1}^{*}}=\frac{\mathrm{TR}}{\mathrm{TR}-\mathrm{T}_{1} \ln (\cos (\alpha))},
$$

where TR is the time between two consecutive excitation pulses and $\alpha$ is the TFE excitation angle. From equation [A2] and [A3] it can be shown that the signal will be more affected when more excitation pulses are applied between the saturation pulse and the acquisition of image $\mathrm{k} 0$. This difference can change with image resolution, profile order, half scan and parallel imaging acquisition factor.

The change in contrast concentration over time can be obtained from the ratio of signal intensity in the baseline situation before contrast injection $\left(\mathrm{S}_{\mathrm{b}}\right)$ to the signal intensity after contrast arrival $\left(\mathrm{S}_{\mathrm{c}}\right)$. By including the signal model described in $\mathrm{Eq}$ [A2] in the ratio it can be demonstrated that the signal relation follows the signal model described by

$$
\frac{\mathrm{S}_{\mathrm{c}}}{\mathrm{S}_{\mathrm{b}}} \mathrm{C}_{1}=\mathrm{e}^{-T E * \Delta R 2^{*}}\left[\epsilon-\left(\epsilon-\left(1-\mathrm{e}^{-R 1_{c} T 0}\right)\right) \mathrm{e}^{-R 1_{c}^{*}(T S-T 0)}\right],
$$

where

$\mathrm{C}_{1}=\epsilon-\left(\epsilon-\left(1-\mathrm{e}^{-R 1_{b} T 0}\right)\right) \mathrm{e}^{-R 1_{b}^{*}(\mathrm{TS}-\mathrm{T} 0)}$ and $\frac{1}{\mathrm{~T} 1_{\mathrm{c}}}=\mathrm{R} 1_{\mathrm{c}}=\mathrm{R} 1_{b}+\Delta \mathrm{R} 1$

Equation A4 states that with knowledge of the baseline T1 obtained by MOLLI sequence [21], the concentration of the contrast agent can be obtained at any time domain point by using the baseline signal as a normalization factor.

Competing interests

JSG is Philips Healthcare employee. The other authors declare that they have no competing interests. 


\section{Authors' contributions}

JSG performed sequence and image analysis development, data processing, phantom experiments, statistical analysis, and drafted the manuscript; RFJ performed experimental animal model, imaging experiments and manuscript drafting; NDN and GLM performed animal imaging experiments and helped in manuscript drafting; VF and BI performed study design, manuscript drafting and critical comments to the analysis of results. All authors read and approved the final manuscript.

\section{Acknowledgments}

This work was supported by a competitive grant from the Ministry of Economy and Competitiveness (MINECO), Fondo Europeo de Desarrollo Regional (FEDER,SAF2013-49663-EXP), Carlos III Institute of Health-Fondo de Investigación Sanitaria (PI13/01979), and FP7-HEALTH-2009 "Cardio Repair European Multidisciplinary Initiative (CARE-MI)". This study forms part of a Master Research Agreement between CNIC and Philips Healthcare. The Spanish Ministry of Economy and Competitiveness and the Pro-CNIC Foundation support the CNIC. Dr. Fernández-Jiménez is a recipient of a Rio Hortega fellowship from the Ministry of Economy and Competitiveness through the Instituto de Salud Carlos III; and has received a FICNIC fellowship from the Fundació Jesús Serra, the Fundación Interhospitalaria de Investigación Cardiovascular, and the CNIC. Nils Nothnagel is a P7-PEOPLE-2011-ITN "Translational Training network on the Cellular and Molecular Bases of Heart Homeostasis and Repair" fellow. Dr. Sánchez-González is an employee of Philips Healthcare. . B.I is supported by the Red de Investigación Cardiovascular (RIC) of the Spanish Ministry of Health (RD 12/0042/0054). Simon Bartlett (CNIC) provided English editing. All other authors have reported that they have no relationships relevant to the contents of this paper to disclose.

\section{Author details}

${ }^{1}$ Philips Healthcare Iberia, Maria de Portugal 1. 28050, Madrid, Spain. ${ }^{2}$ Centro Nacional de Investigaciones Cardiovasculares (CNIC), Melchor Fernandez Almagro 3. 28029, Madrid, Spain. ${ }^{3}$ Hospital Universitario Clínico San Carlos, Madrid, Spain. ${ }^{4}$ The Zena and Michael A. Wiener CVI, Mount Sinai School of Medicine, New York, NY, USA.

\section{Received: 31 October 2014 Accepted: 8 January 2015} Published online: 19 February 2015

\section{References}

1. Blankstein R, Osborne M, Naya M, Waller A, Kim CK, Murthy VL, et al. Cardiac positron emission tomography enhances prognostic assessments of patients with suspected cardiac sarcoidosis. J Am Coll Cardiol. 2014;63:329-36.

2. Chow BJW, Dorbala S, Di Carli MF, Merhige ME, Williams BA, Veledar E, et al. Prognostic value of PET myocardial perfusion imaging in obese patients. JACC Cardiovasc Imaging. 2014;7:278-87.

3. El Aidi H, Adams A, Moons KGM, Den Ruijter HM, Mali WPTM, Doevendans PA, et al. Cardiac Magnetic Resonance Imaging Findings and the Risk of Cardiovascular Events in Patients With Recent Myocardial Infarction or Suspected or Known Coronary Artery Disease: A Systematic Review of Prognostic Studies. J Am Coll Cardiol. 2014;63:1031-45.

4. Morton G, Chiribiri A, Ishida M, Hussain ST, Schuster A, Indermuehle A, et al. Quantification of absolute myocardial perfusion in patients with coronary artery disease: comparison between cardiovascular magnetic resonance and positron emission tomography. J Am Coll Cardiol. 2012;60:1546-55.

5. Wilke N, Simm C, Zhang J, Ellermann J, Ya X, Merkle H, et al. Contrastenhanced first pass myocardial perfusion imaging: correlation between myocardial blood flow in dogs at rest and during hyperemia. Magn Reson Med. 1993:29:485-97.

6. Kraitchman DL, Wilke N, Hexeberg E, Jerosch-Herold M, Wang Y, Parrish TB, et al. Myocardial perfusion and function in dogs with moderate coronary stenosis. Magn Reson Med. 1996;35:771-80.

7. Al-Saadi N, Nagel E, Gross M, Bornstedt A, Schnackenburg B, Klein C, et al. Noninvasive detection of myocardial ischemia from perfusion reserve based on cardiovascular magnetic resonance. Circulation. 2000;101:1379-83.

8. Nagel E, Klein C, Paetsch I, Hettwer S, Schnackenburg B, Wegscheider K, et al. Magnetic resonance perfusion measurements for the noninvasive detection of coronary artery disease. Circulation. 2003;108:432-7.
9. Hsu L-Y, Rhoads KL, Holly JE, Kellman P, Aletras AH, Arai AE. Quantitative myocardial perfusion analysis with a dual-bolus contrast-enhanced first-pass MRI technique in humans. J Magn Reson Imaging. 2006;23:315-22.

10. Christian TF, Rettmann DW, Aletras AH, Liao SL, Taylor JL, Balaban RS, et al. Absolute myocardial perfusion in canines measured by using dual-bolus first-pass MR imaging. Radiology. 2004;232:677-84.

11. Ishida M, Schuster A, Morton G, Chiribiri A, Hussain S, Paul M, et al. Development of a universal dual-bolus injection scheme for the quantitative assessment of myocardial perfusion cardiovascular magnetic resonance. J Cardiovasc Magn Reson. 2011;13:28

12. Sammut E, Zarinabad N, Vianello PF, Chiribiri A. Quantitative Assessment of Perfusion - Where Are We Now? Curr Cardiovasc Imaging Rep. 2014;7:9278.

13. Gatehouse PD, Elkington AG, Ablitt NA, Yang G-Z, Pennell DJ, Firmin DN Accurate assessment of the arterial input function during high-dose myocardial perfusion cardiovascular magnetic resonance. J Magn Reson Imaging. 2004;20:39-45.

14. Kim D, Axel L. Multislice, dual-imaging sequence for increasing the dynamic range of the contrast-enhanced blood signal and CNR of myocardial enhancement at 3 T. J Magn Reson Imaging. 2006;23:81-6.

15. De Bazelaire C, Rofsky NM, Duhamel G, Zhang J, Michaelson MD, George D, et al. Combined T2* and T1 measurements for improved perfusion and permeability studies in high field using dynamic contrast enhancement. Eur Radiol. 2006;16:2083-91.

16. Cernicanu A, Axel L. Theory-based signal calibration with single-point T1 measurements for first-pass quantitative perfusion MRI studies. Acad Radiol. 2006;13:686-93

17. Blüml S, Schad LR, Stepanow B, Lorenz WJ. Spin-lattice relaxation time measurement by means of a TurboFLASH technique. Magn Reson Med. 1993;30:289-95.

18. Fernández-Jiménez R, Sánchez-González J, Aguero J, García-Prieto J, LópezMartín GJ, García-Ruiz JM, et al. Myocardial Edema After Ischemia/ Reperfusion Is Not Stable and Follows a Bimodal Pattern: Advanced Imaging and Histological Tissue Characterization. J Am Coll Cardiol. 2015;65:315-23.

19. García-Prieto J, García-Ruiz JM, Sanz-Rosa D, Pun A, García-Alvarez A, Davidson SM, et al. $\beta 3$ adrenergic receptor selective stimulation during ischemia/reperfusion improves cardiac function in translational models through inhibition of mPTP opening in cardiomyocytes. Basic Res Cardiol. 2014;109:422.

20. Jensen JH, Chandra R. NMR relaxation in tissues with weak magnetic inhomogeneities. Magn Reson Med. 2000;44:144-56.

21. Messroghli DR, Plein S, Higgins DM, Walters K, Jones TR, Ridgway JP, et al. Human myocardium: single-breath-hold MR T1 mapping with high spatial resolution-reproducibility study. Radiology. 2006;238:1004-12.

22. Van Osch MJP, Vonken EP, Viergever M, van der Grond J, Bakker CJG. Measuring the arterial input function with gradient echo sequences. Magn Reson Med. 2003;49:1067-76.

23. Kalavagunta C, Metzger GJ. A field comparison of $r 1$ and $r 2$ * relaxivities of Gd-DTPA in aqueous solution and whole blood : 3 T versus $7 \mathrm{~T}$. Proc ISMRM. 2010;18:4990.

24. Kim TH, Pack NA, Chen L, Dibella EVR. Quantification of myocardia perfusion using CMR with a radial data acquisition: comparison with a dual-bolus method. J Cardiovasc Magn Reson. 2010;12:1-8.

25. Pintaske J, Martirosian P, Graf H, Erb G, Lodemann K-P, Claussen CD, et al. Relaxivity of Gadopentetate Dimeglumine (Magnevist), Gadobutrol (Gadovist), and Gadobenate Dimeglumine (MultiHance) in human blood plasma at 0.2, 1.5, and 3 Tesla. Invest Radiol. 2006:41:213-21.

26. Gatehouse P, Lyne J, Smith G, Pennell D, Firmin D. T2* effects in the dual-sequence method for high-dose first-pass myocardial perfusion. J Magn Reson Imaging. 2006;24:1168-71.

27. Chiribiri A, Schuster A, Ishida M, Hautvast G, Zarinabad N, Morton G, et al. Perfusion phantom: An efficient and reproducible method to simulate myocardial first-pass perfusion measurements with cardiovascular magnetic resonance. Magn Reson Med. 2013;69:698-707.

28. Hsu L-Y, Groves DW, Aletras AH, Kellman P, Arai AE. A quantitative pixel-wise measurement of myocardial blood flow by contrast-enhanced first-pass CMR perfusion imaging: microsphere validation in dogs and feasibility study in humans. JACC Cardiovasc Imaging. 2012;5:154-66.

29. Kellman P, Aletras AH, Hsu L-Y, McVeigh ER, Arai AE. T2* measurement during first-pass contrast-enhanced cardiac perfusion imaging. Magn Reson Med. 2006:56:1132-4 